\title{
Dry Powder Deposition and Compaction for Functionally Graded Ceramics
}

\author{
Zachary N. Wing ${ }^{\star \dagger}$ and John W. Halloran ${ }^{\star \star}$ \\ Department of Materials Science Engineering, University of Michigan, Ann Arbor, Michigan 48109
}

\begin{abstract}
Functionally graded ceramics, with property variations realized via local compositional changes or porosity, are fabricated by powder deposition and compaction. A removable fixture is used to control the location of dry powders to produce a macroscopic powder array with 2-D or 3-D compositional variations. A new method is presented that relies on traditional powder pressing techniques to create spatial variation in composition and density. The fixture is removed before conventional powder compaction. This dry powder deposition and compaction method is capable of producing large monolithic bodies with functional gradients. This method produces designs with $3 \mathrm{~mm}$ discrete regions or pixels that have a normalized positional tolerance of $0.6 \%$ for a $39 \mathrm{~mm}$ substrate size. The method is used to realize a UHF antenna substrate with spatially variable dielectric properties.
\end{abstract}

\section{Introduction}

$\mathrm{P}$ OWDER processing is the most efficient method for mass producing ceramic components. The process itself is simple and consists of three basic steps: powder filling a die, compaction of the powder, and ejection of the green compact. Production rates for ceramics greater than 5000 parts per minute (ppm) are possible. ${ }^{1}$ Pharmaceutical powder pressing has reported rates $>15000 \mathrm{ppm} .^{2}$ Dry powder methods are advantageous because their binder content is small compared with thermoplastic methods, which can have substantial binder contents ( $\sim 50 \mathrm{~V} \%)$. Therefore, much larger parts can be made via dry powder methods without binder burnout limitations. However, powderpressed ceramics typically have a uniform composition. Complex material variations are hard to achieve using traditional powder pressing due to the difficulty in controlling the location of dry powder. Layered structures, which have horizontal variations normal to the compaction direction, have been realized previously, but variations parallel to the axis of compaction are uncommon. Recently, we have shown that distinct vertical interfaces can be maintained during uniaxial compaction, if the dry powders were carefully located before compaction. Powder location was controlled using a removable divider placed vertically within a die to maintain a 1-D texture. This was done to create monolithic dielectrics with textures $\sim 2 \mathrm{~cm}$. This was demonstrated with compositionally variable calcium magnesium titanates (CMTs) ceramics for dielectrically loaded antennas. ${ }^{3}$ The compositional and dielectric variations were characterized by scanning microwave microscopy. ${ }^{4}$ In this work, we extend this method to achieve 2-D and 3-D compositional variations using a removable fixture to control the deposition of powder parallel to the compaction axis in a thick bed.

The controlled deposition of dry powders in well-controlled 2-D patterns is not new. Rather, it is an ancient art. Sand paint-

K. Faber-contributing editor

\footnotetext{
Manuscript No. 21571. Received March 9, 2006; approved June 22, 2006.

${ }^{*}$ Member of the American Ceramic Society.

${ }^{* \star}$ Fellow of the American Ceramic Society.

Author to whom correspondence should be addressed. e-mail: wingzach@umich.edu
}

ing, where colored sand is ritualistically deposited, is a ceremonial practice used by Tibetan Buddhist monks and by Navajos. The Navajo method dispenses sand between the thumb and index finger. ${ }^{5,6}$ Tibetan monks dispense powder through ritual tapping of ceremonial funnels. ${ }^{7}$ Sand art in 3-D is available as sand vases in many gift shops. The production of these works of art is a great exercise in skill and patience, but hand deposition has been replaced by computer-controlled deposition. At the 2004 SIGGRAPH Conference of the Association for Computing Machinery, artist Dan Collins demonstrated "Sand Printer," a $7.7 \mathrm{~m} \times 4.6 \mathrm{~m}$ machine that produced sand paintings from $12 \mathrm{~mm}$ pixels of sand. ${ }^{8}$ Single-layer patterns of colored glass beads have been deposited with computer-controlled capillaries as a model for multi-material beds for scanning laser sintering. ${ }^{9}$ Yang and Evans ${ }^{10}$ have demonstrated multi-component powder dispensing for three-dimensional functional gradients and 3 -D alumina-metal objects, ${ }^{11}$ using acoustic control of powder flow in capillaries. ${ }^{12}$

However, deposition of multi-material powder beds is not sufficient for many functional gradient ceramics. Loose powders deposit at solids loadings near their tap density, typically in the range of $30 \%$ of theoretical density. Such packing densities are not high enough to subsequently sinter the ceramic to near full density. Post-deposition consolidation is necessary to increase the density to achieve a sinterable green body.

The purpose of this work is to present a new method for creating 2-D and 3-D functional gradient ceramics as spatially variable dielectrics in which regions of variation are discretized into $3 \mathrm{~mm}$ square pixels. The process of dry powder deposition and compaction can be applied with multiple dielectrics having similar firing behavior and chemical stability, so that they can be cofired to produce dense ceramics with useful dielectric function. Spatially variable properties are important for novel electromagnetic devices ${ }^{13,14}$ (filters, antennas, etc.) that exploit geometry and energy feeds to maximize their performance. The dielectric material distribution can be very complex, ${ }^{15}$ requiring hundreds of discrete regions with different dielectric properties.

\section{Experimental Procedure}

\section{(1) Dry Powder Deposition and Compaction (DPDC) Process Description}

The DPDC method controls the location of loose powder before compaction. Figure 1 illustrates the process. The process is composed of three steps: powder deposition, fixture extraction, and compaction. Powder is deposited inside the wells of a windowpane fixture consisting of many small pixels separated by thin walls (Step 1). We find it convenient to fill the wells manually using a small spatula, using the vibration of the spatula and its degree of inclination to control the powder flow. Each well may hold a compositionally different (but compatible) powder. Once the wells are filled according to the desired design, the fixture is extracted (Step 2) by carefully lifting it out with forceps. Extraction causes very little distortion. Finally, the patterned ensemble of loose powders is consolidated by conventional compaction (Step 3), creating a green compact with a variable composition design. Full 3-D compositional variation can be 

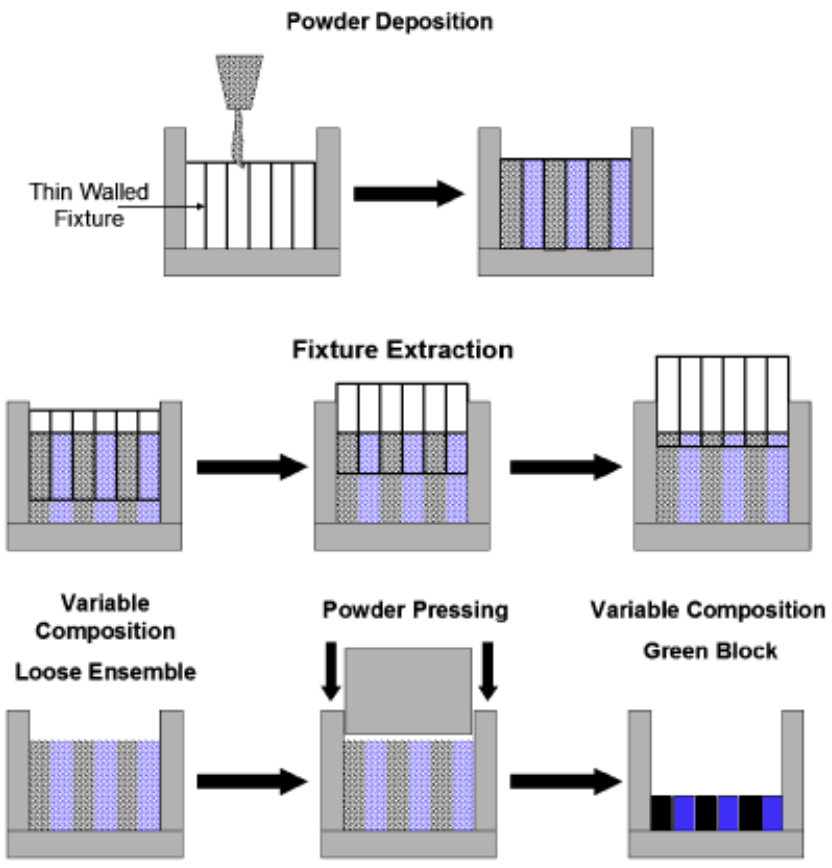

Fig. 1. Dry powder deposition and compaction process steps.

achieved by depositing powders in two stages (i.e., filling a well halfway). A two-stage fill creates layers within each well.

\section{(2) Materials}

We used commercial spray-dried powders of CMTs, a technologically important family of dielectrics of interest for microwave applications due to the wide range of dielectric constants available. These powders are three-phase mixtures of $\mathrm{CaTiO}_{3}$, $\mathrm{MgTiO}_{3}$, and $\mathrm{Mg}_{2} \mathrm{TiO}_{4}$ that are thermodynamically stable. ${ }^{16}$ Typically, higher $\mathbf{k}$ CMTs have a higher fraction of $\mathrm{CaTiO}_{3}$. Three commercial powders from the CMT family were selected, one with a dielectric constant of 20, 70, and 115 (CMT 20, 70, and 115, Trans-Tech Inc., Adamstown, MD). These powders have a mean particle size of $1-2 \mathrm{~m}$ and have spray-dried agglomerates 15-180 m, as shown in Fig. 2 for the CMT 20 and CMT 70. The compaction response of these two powders was characterized by measuring density versus compaction pressure with a displacement-controlled mechanical testing machine, and was found to be similar within $2 \%$. An antenna substrate was fabricated according to an optimized topology design ${ }^{15}$ using the CMT 70, CMT 20, and a material with a dielectric constant of 15 , prepared by inducing 25 vol $\%$ spherical pores with glassy carbon microspheres (Sigradur K - $15 \mu \mathrm{m}$, HTW, Thierhaupten, Germany). Porosity is known to change the dielectric constant. ${ }^{17,18}$ Dielectric constant variations with porosity in this system are reported in detail elsewhere. ${ }^{19}$

\section{(3) Processing}

A fixture to control powder location was created consisting of $3 \mathrm{~mm}$ square pixels arranged in a $12 \times 13$ rectangular grid. Fixture walls were $0.5 \mathrm{~mm}$ thick and the height was $15 \mathrm{~mm}$. A CAD file is used to create a stereo lithographic file (STL), which was exported to a rapid prototyping machine (SLA-250, 3D Systems, Valencia, CA) to produce a rigid plastic fixture using a solid imaging photopolymer (Somos 8110, DSMSomos, New Castle, DE). The fixture is created $0.4 \mathrm{~mm}$ smaller than the die to ensure smooth extraction. After extracting the fixture, samples were compacted uniaxially inside a lubricated steel die $(47 \mathrm{~mm} \times 44 \mathrm{~mm})$ at $50 \mathrm{MPa}$ with the pressure held for $5 \mathrm{~min}$. Sintering was conducted in air using a heating rate of $10^{\circ} \mathrm{C} / \mathrm{min}$ to a peak temperature of $1360^{\circ} \mathrm{C}$, with a $2-\mathrm{h}$ dwell and a cooling rate of $5^{\circ} \mathrm{C} / \mathrm{min}$. The sintered materials were at least $95 \%$ dense, as judged by quantitative stereology, except for those areas with porogen-induced porosity.

\section{(4) Characterization of Dimensional Fidelity}

Samples were characterized to assess the process fidelity and investigate the development of distortions during compaction. Samples were produced using CMT 20 and 70 arranged in a periodic array of square pixels. Owing to variations in composition, there is optical contrast between the two dielectric powders. A periodic array was selected to provide numerous points for quantitative measurements. Samples were studied during each processing step. Analyzed samples included the loose powder ensemble after fixture extraction, compacted green body, and sintered specimens.

Loose powder (as deposited) and compacted powder samples were vacuum infiltrated with epoxy. The epoxy infiltration imparts strength to the weak green body so it can be sectioned. It is assumed that the infiltration process does not disturb the powder position. This was performed using a low-viscosity "Spurs" medium (Low Viscosity Kit 18300-4221, Pelco International, Redding, CA). Samples were held under vacuum for $10 \mathrm{~min}$. After degassing the samples, infiltration of the epoxy was performed at 25 mbar (Epouvac, Struers Inc., Cleveland, OH).

All samples were analyzed in the $x-y$ plane at multiple heights. Sections were achieved by grinding with a 180-grit $\mathrm{SiC}$ paper. Optical images of each section were captured using a digital camera. Dimensions were obtained through digital imaging measurements.

Process fidelity was characterized by comparing the theoretical location of vertices with the actual location. Ten points or pixel vertices were analyzed at multiple heights within a sample. The absolute distance from a sample corner to the point of interest is used as the fidelity metric. Distortions were characterized by the $x-y$ dimensions of pixels as a function of height. Fifteen pixels were analyzed at each height.
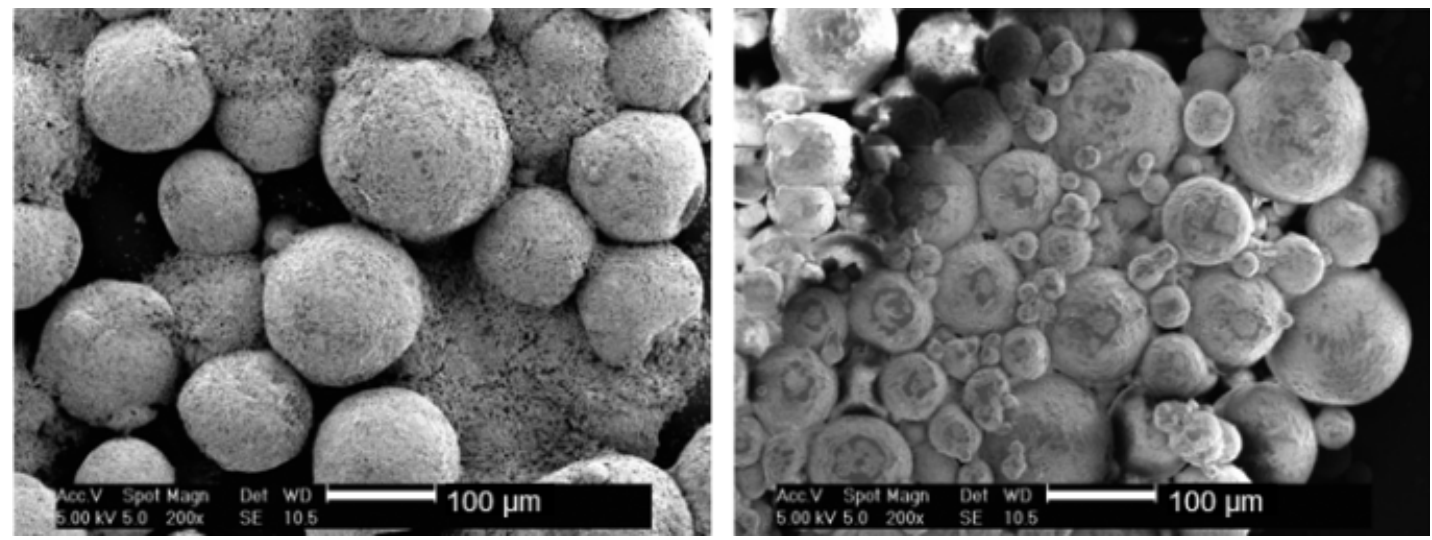

Fig. 2. Scanning electron microscopy image of spray-dried powders (left) CMT 20 (right) CMT 70. 


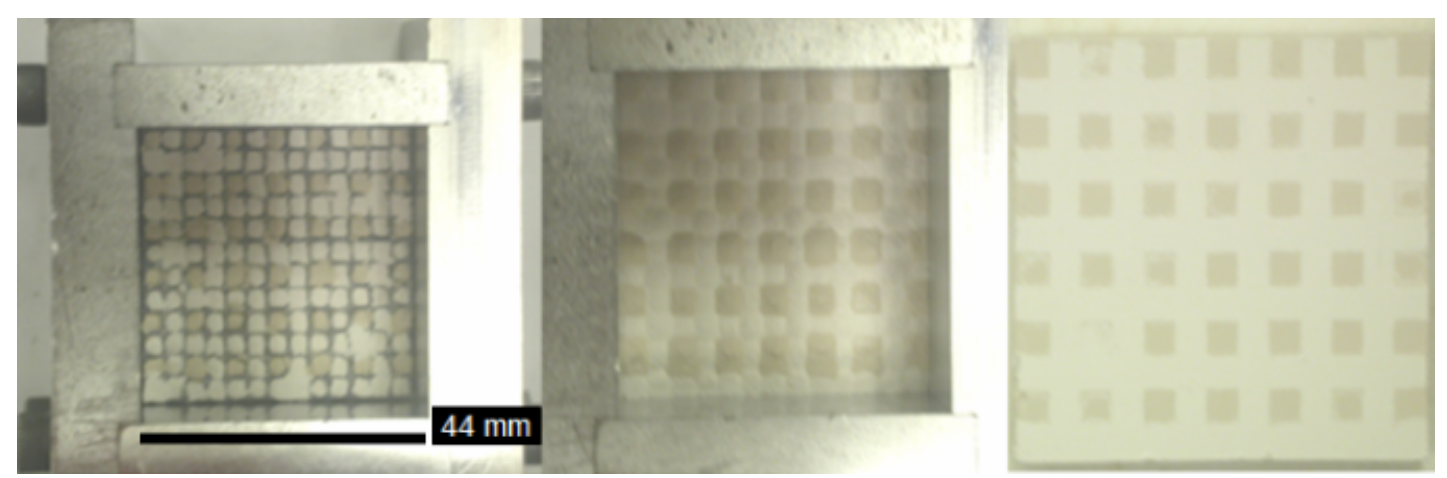

Fig. 3. Formation of the green body. (Left) Filled wells in die (middle) after fixture extraction (right) after powder compaction (die bottom).

\section{Results and Discussion}

\section{(1) Process Demonstration}

The first example is a simple checkerboard pattern consisting of $3 \mathrm{~mm}$ pixels of CMT 20 and CMT 70 dielectric. These were produced by using a fine spatula to fill alternately a windowpane fixture so that one well had CMT 20 powder and the adjacent well had CMT 70 powder. Figure 3 (left) shows the windowpane fixture loaded in a checkerboard fashion. The filled fixture is inserted into the compaction die and the plastic fixture is carefully extracted by lifting it out. The top of the loose powder after extraction appears in Fig. 3 (middle). At this stage, it shows some distortions due to an uneven fill in the fixture wells, but it is limited to a thin layer. The compaction ram is inserted into the die and the powder is pressed at $50 \mathrm{MPa}$, resulting in a pressed density of $56 \%$. Producing a $5 \mathrm{~mm}$ green compact requires $\sim 5$ $\mathrm{mm}$ of vertical travel, but this occurs with very little transverse distortion. Figure 3 (right) shows good fidelity on the die bottom of the green compact.

To illustrate the type of designs that have been executed, four examples are presented. The checkerboard design after sintering is shown in Fig. 4(a). It is a $39 \mathrm{~mm} \times 33 \mathrm{~mm}$ rectangular plate, $4 \mathrm{~mm}$ thick, of a nominally dense ceramic with 156 alternating pixels. Figure 4(b) shows other examples from the same material where the CMT 20 material in light contrast forms an "M" design. A three-material design is shown in Fig. 4(c), involving CMT materials with a dielectric constant of 20 (light), 70 (medium), and 115 (dark color). Finally, Fig. 4(d) illustrates a bilayer checkerboard design, demonstrating 3-D compositional variation.

\section{(2) Process Characterization}

(A) Pixel Dimensions: The fixture used to separate powders was analyzed to determine its dimensions and predict the final dimensions of a given design. Although the fixture was designed to consist of uniform cells, it is slightly non-uniform. Cells and cell walls contain some variation due to the geometrical requirements of the rectangular die (in which it must fit) and to variations in the stereo lithography fabrication. The average interior dimensions of a cell showed a slight asymmetry of $3.10 \mathrm{~mm}(x)$ and $3.07 \mathrm{~mm}(y)$. Cell walls also showed an asymmetry with an average $x$ and $y$ thickness of 0.46 and $0.55 \mathrm{~mm}$, respectively. Thus, cell wall effects are significant and must be accounted for to predict the final size after fixture extraction. In addition to cell wall effects, other sources of uncertainty are: variations in the fixture dimensions, fixture movement, and large agglomerates within the powder. After accounting for these effects, the expected $x-y$ cell size in the green body was $3.56 \pm 0.110 \mathrm{~mm} \times 3.62 \pm 0.110 \mathrm{~mm}$, respectively. Measurements were performed on the 15 pixels shown in Fig. 5 (left).

The average pixel dimensions were compared against the expected dimensions. A relative height of zero corresponds to

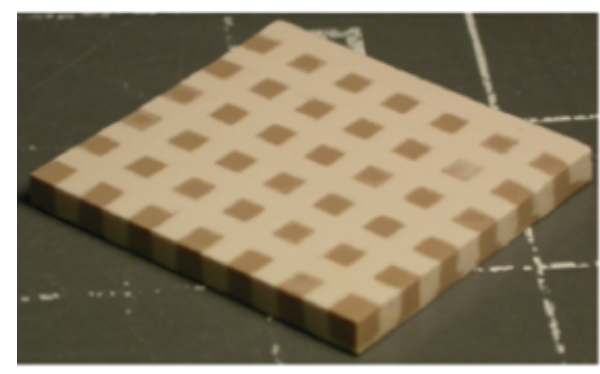

(a)

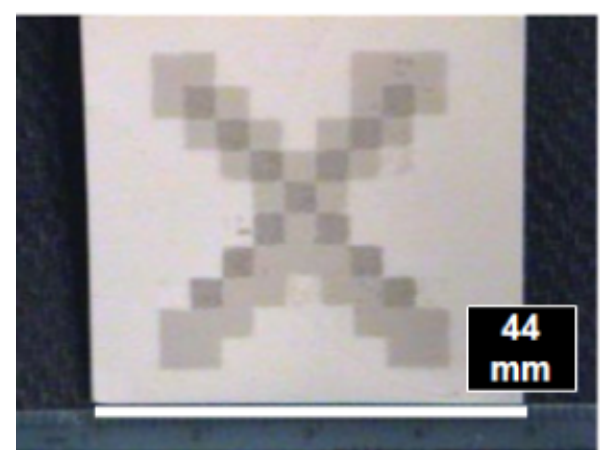

(b)

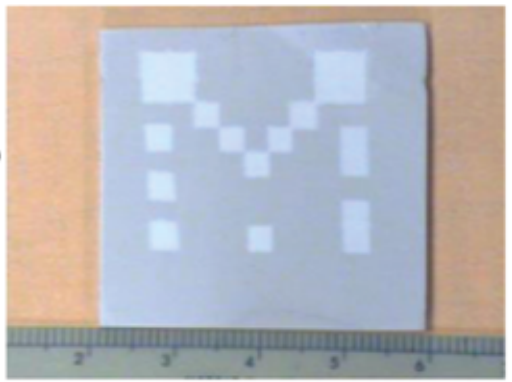

(c) (d)

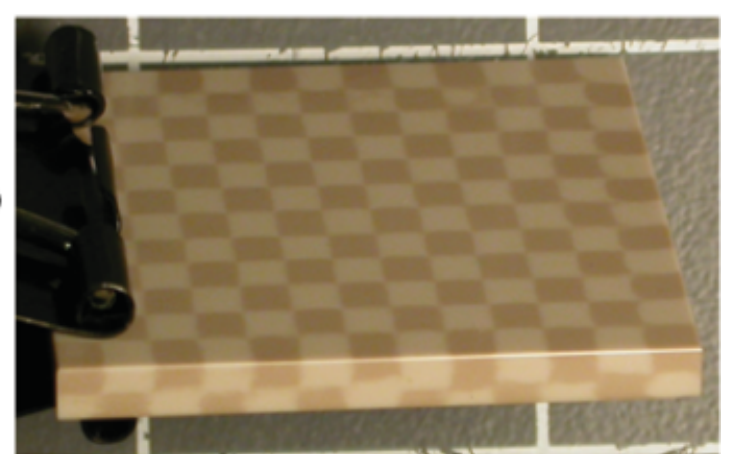

Fig. 4. Demonstration samples of sintered samples. All $39 \mathrm{~mm} \times 33 \mathrm{~mm}$ (pixels $3 \mathrm{~mm}$ square). (a) 2-D checkerboard, 2 materials (light CMT 20, dark CMT 70). (b) 2-D "M" design, 2 materials (light CMT 20, dark CMT 70). (c) 2-D “double X", 3 materials (light CMT 20, medium CMT 70, dark CMT 115). (d) 3-D Checkerboard, 2 materials (light CMT 20, dark CMT 70). 

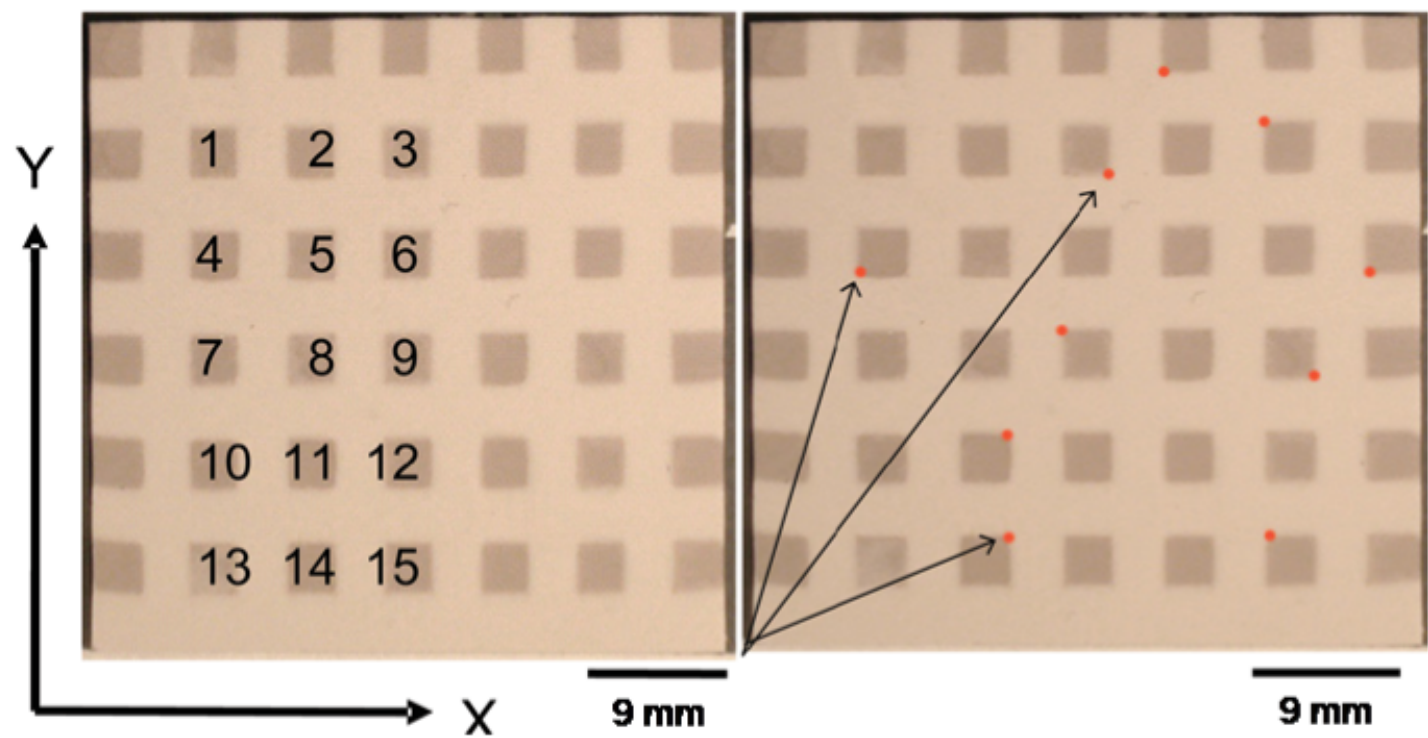

Fig. 5. (Left) Pixels measured for $x-y$ analysis (right) points for fidelity analysis.

the sample surface touching the die bottom and a height of 1.0 corresponds to the sample surface touching the moving piston. In the green powder compact, the expected dimensions are the same as the loose powder body because it is assumed that powder moves uniaxially during compaction. It is assumed that epoxy infiltration does not disturb the powder orientation in the green bodies.

In the loose powder samples, the average $x$ dimension was $3.48 \pm 0.061 \mathrm{~mm}$ and the average $y$ dimension was $3.57 \pm 0.061$ $\mathrm{mm}$ throughout the sample. The average dimensions for the compacted green body were $3.57 \pm 0.061$ and $3.62 \pm 0.061 \mathrm{~mm}$ in $x$ and $y$, respectively. High distortions in pixel size were observed at the highest relative heights (above 0.74 ). This is not surprising because the top surface of the loose powder undergoes a large uniaxial displacement. In addition, the extraction of the fixture leaves the loose powder surface with miniature "mounds" as the powder is not self-leveling like a fluid. Hence, high relative height distortions are not surprising. Average values exclude measurements $>0.75$ relative height in the green samples.
Similar measurements were performed with sintered samples. The expected dimensions are smaller than the previous samples due to densification shrinkage. There is a 3\% mismatch shrinkage (linear) between CMT $20(15 \%)$ and CMT 70 (18\%). Thus, the average shrinkage, $16.5 \%$, was used to estimate the final pixel size, which was determined to be $2.92 \pm 0.09$ and $2.97 \pm 0.09$ $\mathrm{mm}$, for $x$ and $y$, respectively. The expected values are in good agreement with the measured values. The average sintered pixel size is $2.95 \pm 0.05$ and $3.01 \pm 0.05 \mathrm{~mm}$ in $x$ and $y$, respectively.

Overall, there is good agreement with the expected values in both the green and sintered state. However, there does appear to be a weak trend showing increasing pixel size with increasing sample height (moving toward the piston surface). Comparison of the values at heights of 0 and 0.74 shows an expansion of $4.07 \%$ in $x$ and $3.26 \%$ in $y$. The expansion is due to the misfit between the fixture and the die. If the fixture had fit tightly within the die, this would not be observed. However, to ensure smooth fixture extraction, some "free space" between the fixture and die remains. During the die extraction, powder flows into
-Expansion is due to misfit between the fixtme and die

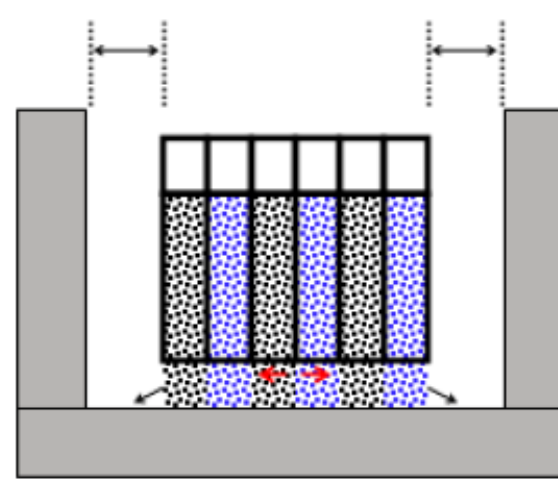

-Small displacements at die bottom

\section{- Ontward flow is greater near the die top.}
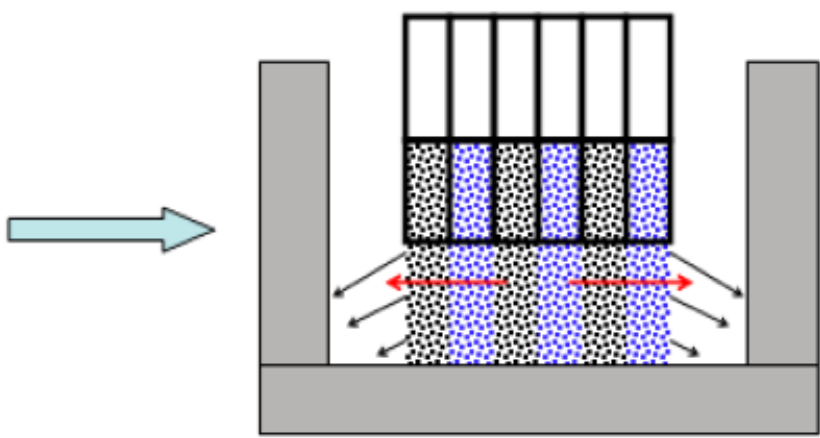

- Larger displacements at die top

Fig. 6. Fixture-die misfit leads to pixel expansion with increasing height. 


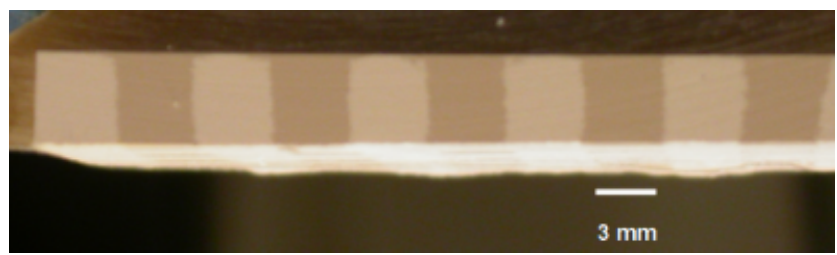

Fig. 7. Cross section of green powder compact.

the extra free space accounting for pixel expansion. Because of powder flow (not fluid flow), the powder at higher relative heights (closer to the top of the loose powder) will flow down and outward toward the free space. This is illustrated in Fig. 6.

Figure 7 shows a cross section of a powder-compacted sample. The right pixel shows evidence of a flow distortion. It was also determined that variations in loose powder height ("humps") also led to a thin layer of distortions. Figure 8 shows this observed distortion. Distortions due to pressure gradients and die wall effects were inconclusive due to overall outward flow expansion.

(B) Vertex Location: In the previous section, the size of the features (pixels) was analyzed. This section studies the position of those features and is important to determine how well a design is replicated in the sintered body. The positions of the vertices for 10 pixels were measured (Fig. 5-right) with respect to a sample corner and compared with the expected distance.

Figure 9 shows the measured vertices position compared with the theoretical position for two sintered samples. The graph shows that the deviations from the expected values are small and that the vertices position is well described. Data points from both samples overlap and have an uncertainty of $\pm 0.056 \mathrm{~mm}$ $(56 \mu \mathrm{m})$. Throughout both samples, the location of a given vertex deviates from the theoretical position by $0.220 \mathrm{~mm}$ or $220 \mu \mathrm{m}$. Normalizing $220 \mu \mathrm{m}$ by the largest substrate dimension (39 $\mathrm{mm}$ ) yields a tolerance of $0.6 \%$.

A more detailed illustration of the deviations between the measured vertex location and theoretical vertex location is shown in Fig. 10. The deviation is defined as the difference between the theoretical distance from origin $\left(D_{\mathrm{Th}}\right)$ and the measured distance $\left(D_{\mathrm{M}}\right)$. The two data sets show an increasing deviation from theoretical values with increasing distance from the origin. Sample no. 2 shows similar behavior but with a noticeable shift with respect to sample no. 1. The two data sets were fit using linear regression. Table I summarizes the linear regression analysis for two sintered samples at three vertical locations, or relative heights of 0.00 (bottom at the fixed platen), 0.24 (about one quarter of the thickness), and 0.50 (center). The slope is referred to as the bias within the sample. It represents the propagation or drift of errors that occur with increasing

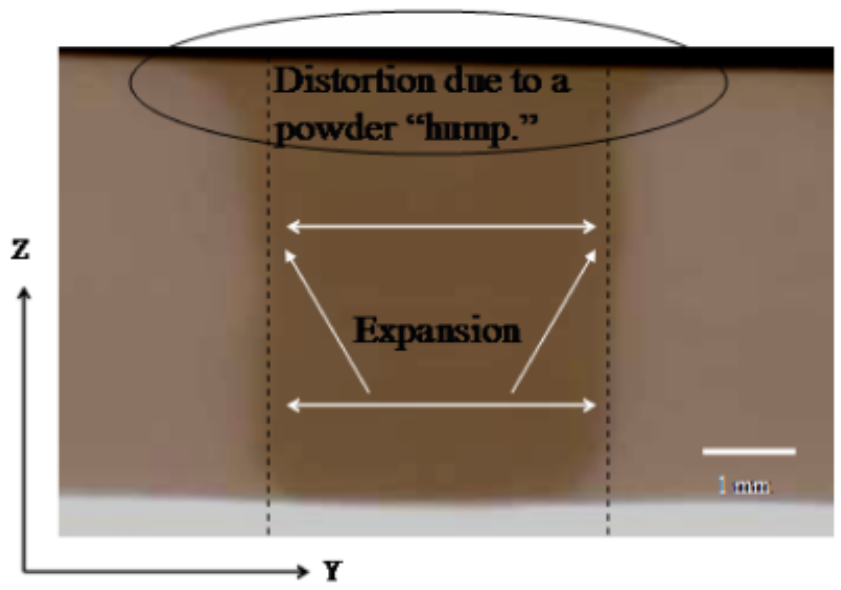

Fig. 8. Small pixel expansion with increasing height. The top of the sample shows near-surface distortion due to uneven loose powder before compaction.

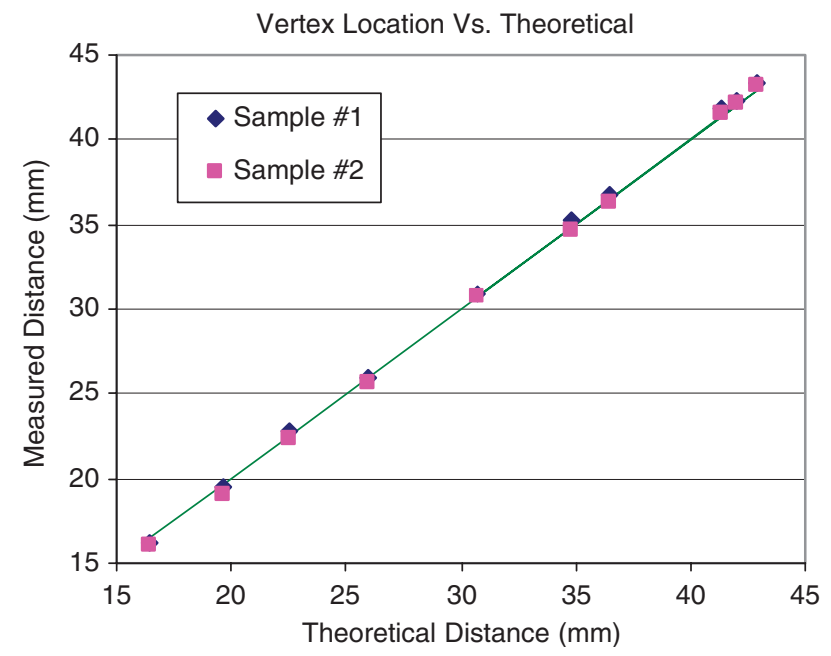

Fig. 9. Measured vertices distance from origin at 0.5 relative sample height. Samples no. 1 and no. 2 show good correlation.

distance from origin. This error is likely due to uncertainties in the local shrinkage behavior. The average bias across each sample is $-0.017 \mathrm{~mm} / \mathrm{mm}$.

The intercept values are referred to as the offset values. It represents a shift of the entire body with respect to the origin. This arises due to the misfit distance between the die and the fixture. If the fixture is located in a different location during extraction, it will cause all vertices to shift. The average offset difference between the samples is $0.28 \mathrm{~mm}$. The last column in Table I shows the residual sum of squares. It is an indication of the scatter between the regression fit and the measured values. For both samples, the regression fit is within $0.1 \mathrm{~mm}$ or $100 \mu \mathrm{m}$, suggesting a good fit. The magnitude of the scatter is on the order of the size of the spray-dried granules, which themselves are the primitive units from which the design is built.

\section{(3) Application}

Figure 11 shows one segment of a 20 -segment substrate for an advanced UHF antenna substrate, designed by Kiziltas et al. ${ }^{16}$ via numerical optimization of the dielectric distribution to achieve a target bandwidth. Figure 11 (left) shows one corner of the optimal design, illustrated as an array of 156 pixels having dielectric constant values of 70 (red), 20 (blue), and 15 (yellow). The actual design was readily realized using DPDC. After compaction, the green body in Fig. 11 (middle) showed good correlation with the design, where the CMT 70 material is dark

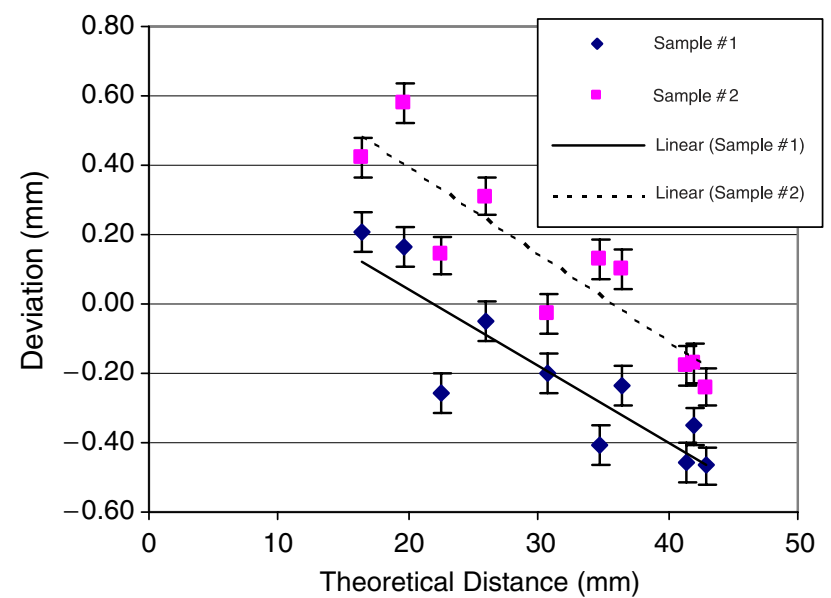

Fig. 10. Deviation $\left(D_{\mathrm{Th}}-D_{\mathrm{M}}\right)$ versus theoretical distance for 0.5 relative sample height. Linear regression lines are fit for two sintered samples. 
Table I. Linear Regression Results for Sintered Sample Vertices Deviation

\begin{tabular}{lccc}
\hline & Bias $(\mathrm{mm} / \mathrm{mm})$ & Offset $(\mathrm{mm})$ & Rss $(\mathrm{mm})$ \\
\hline Sample no. 1 & & & \\
0.00 & -0.015 & 0.28 & 0.09 \\
0.24 & -0.015 & 0.41 & 0.04 \\
0.50 & -0.022 & 0.49 & 0.11 \\
Sample no. 2 & & & \\
0.00 & -0.008 & 0.40 & 0.09 \\
0.24 & -0.019 & 0.73 & 0.15 \\
0.50 & -0.025 & 0.89 & 0.13 \\
\hline
\end{tabular}

beige, the CMT 20 is light beige, whereas the material with carbon microsphere porogens is black. Pixels were $3.6 \mathrm{~mm}$ square. Deviations from the actual design are typically surface effects or due to human error. The sintered body shown in Fig. 11 (right) agrees well with the design, although there is little optical contrast between the regions of dielectric contrast 20 and 15. Sintered pixels were $3 \mathrm{~mm}$ square. Figure 12 shows secondary electron images of the as-fired surface of the UHF substrate. On the left is a higher magnification view of a pixel with dielectric constant 70, showing the typical grain size for this powder sintered under these conditions, with a grain size of 3-4 $\mu \mathrm{m}$ and a residual porosity of about $5 \%$. On the right is a lower magnification view of several pixels. There is little contrast between the pixel with dielectric constant 70 and 20 . However, the pixels with dielectric constant 15 are distinct due to the induced porosity. Note that the glassy carbon porogen was mixed with the spraydried granules, so much of the induced porosity is inter-granular. The complete experimental UHF substrate required $60 \mathrm{seg}$ ments similar to Fig. 11 (although with different designs) requiring $\sim 17000$ pixels. The electromagnetic performance of these spiral antennas with this substrate is presented elsewhere. ${ }^{15}$

\section{(4) Process Summary (Limitations and Advantages)}

Limitations for DPDC include fixture geometry, deposition method, and powder flow. The segregating fixture should be a thin-walled structure. This is necessary to minimize flow between cells and impacts the final resolution. Powder delivery is performed using a hand-controlled spatula. The smallest hole that one can reliably fill (with minimal overflow in an undesired cell) is $3 \mathrm{~mm}$. The key requirement for successful DPDC is powder flow characteristics. However, powder handling has its difficulties due to cohesion. Particle cohesion due to van der Waals forces is a problem for fine particles $<30 \mu \mathrm{m} .{ }^{20}$ It is essential that the working powders are free flowing. The spray-dried powders presented (100 $\mu \mathrm{m}$ agglomerates) enable easy deposition and ensure that the spatial location is maintained during fixture removal.

The presented process is not currently capable of complex geometries, but is capable of producing complex compositional and porosity gradients. It is a relatively rapid fabrication method than can achieve large geometries (30 min for $4.7 \mathrm{~cm} \times 4.4$ $\mathrm{cm} \times 1 \mathrm{~cm}$ green body). The process is also material insensitive (providing all powders have co-fire compatibility and are free flowing). This method could be scaled up to provide economical mass production of a given design using a set of dedicated powder shoes for a given fixture well. Although the process describes creating a "pixelated" design, the process could be adapted to "non-pixelated" designs.

DPDC can be readily adapted for computerized powder deposition, using the technique of Zhang and Evans for example, which would enable automated prototype fabrication. For mass production of a fixed design, a suitable modified powder shoe
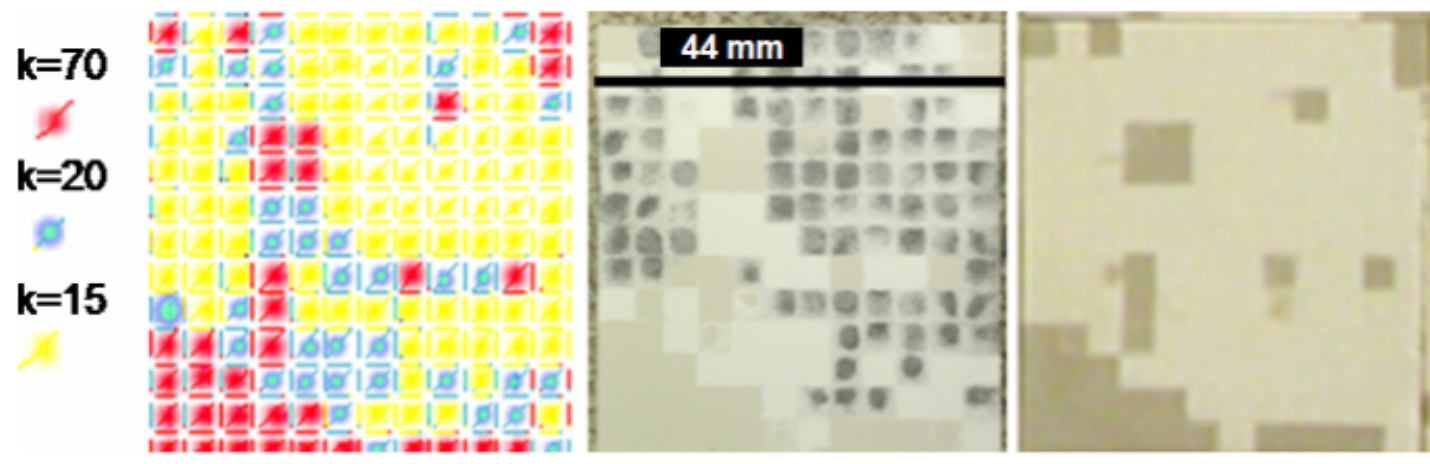

Fig. 11. Optimal dielectric distribution (left) design (middle) dry powder deposition and compaction (DPDC) green body (right) sintered body. Pixels are $3.6 \mathrm{~mm}$ in the green body and $3 \mathrm{~mm}$ in the sintered body.
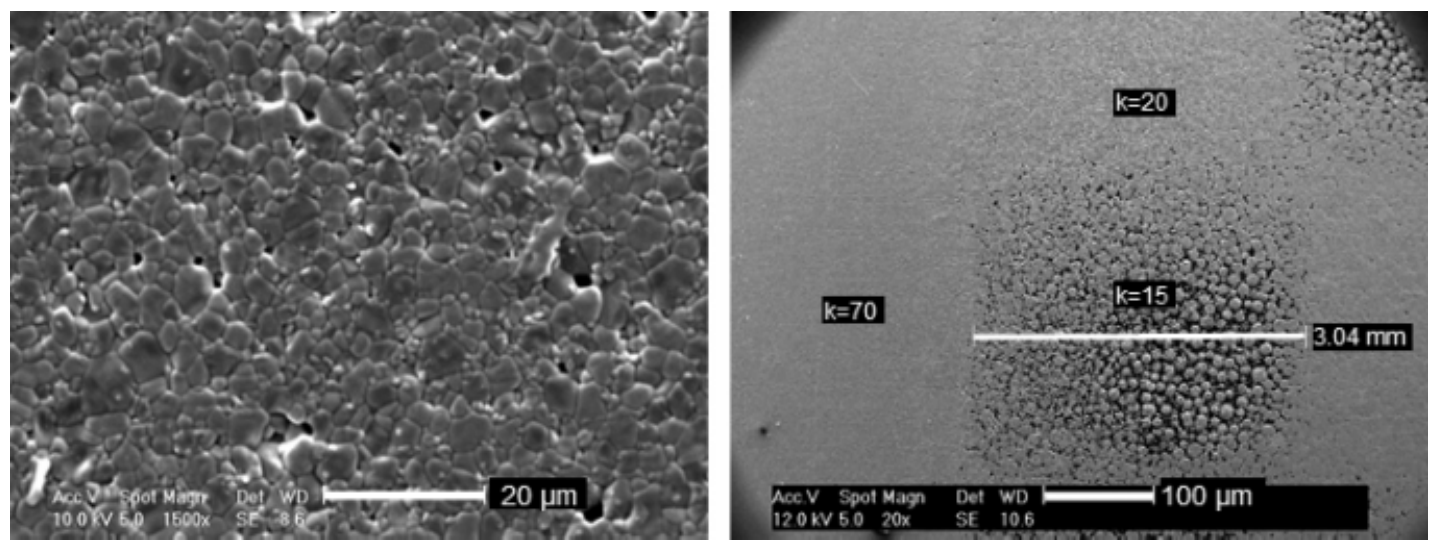

Fig. 12. Secondary electron images of UHF substrate. (Left) As-fired surface of pixel with a dielectric constant of 70. (Right) Wide-angle view showing $3 \mathrm{~mm}$ pixels. 
could rapidly fill a compaction die with the appropriate spatially variable powders.

\section{Summary and Conclusion}

A new method of creating spatially variable dielectrics is presented based on dry powder deposition. This method is based on controlling the location of loose powder before compaction. DPDC capabilities were demonstrated by fabricating several simple textures. Characterization of the process showed that the dimensions of test pixels agreed well with the theoretical dimensions during each step of the process. Fidelity in the final sintered body showed that points were within $220 \mu \mathrm{m}$ of their expected position, which is $0.6 \%$ of the substrate size. Finally, a complex dielectric texturization is realized, monolithically, that exhibits a spatial variation in both composition and density. Refinements to the fixture and automation of the powder deposition phase will allow similar gradients in composition and density but with discretized regions of $1 \mathrm{~mm}$ or less.

\section{References}

${ }^{1}$ J. S. Reed, Introduction to the Principles of Ceramic Processing. Wiley, New York, 1988.

${ }^{2}$ N. G. Lordi and A. M. Cuitino, "Compaction of pharmaceuticals," MRS Bulletin, 22 [12] 34-37 (Dec. 1997).

${ }^{3}$ D. Psychoudakis, J. L. Volakis, Z. N. Wing, S. K. Pillai, and J. W. Halloran, "Enhancing UHF Antenna Functionality Through Dielectric Inclusions and Texturization," IEEE Trans. Antennas Propagation, 54 [2, Part 1] 317-29 (2006).

${ }^{4}$ Z. N. Wing, J. W. Halloran, Q. Zhang, and P. J. McGinn, "Variable Dielectrics in the CMT System Characterized with SMM," J. Am. Ceram. Soc., 89 [5] 1610-4 (2006).

${ }^{5} \mathrm{G}$. Witherspoon, Language and Art in the Navajo Universe. University of Michigan Press, Michigan, 1977.
${ }^{6}$ S. Page and J. Page, Navajo. Harry N. Abrams Inc. Publishers, New York, NY, 1995.

${ }^{7}$ W. Herzog, "Wheel of Time"; Werner Herzog Filmproduction, April 16, 2004 ${ }^{8} \mathrm{http}$ //en.wikipedia.org/wiki/Image:SIGGRAPH04-SandPrinting.jpg

${ }^{9}$ P. Kumar, J. K. Santosa, E. Beck, and S. Das, "Direct-Write Deposition of Fine Powders Through Miniature Hopper-Nozzles for Multi-Material Solid Freeform Fabrication," Rapid Prototyping J., 10 [1] 14-23 (2004).

${ }^{10}$ S. Yang and J. R. G. Evans, "A Multi-Component Powder Dispensing System for Three Dimensional Functional Gradients," Mater. Sci. Eng. A, 379, 351-9 (2004).

${ }^{11}$ M. M. Mohebi, S. Yang, and J. R. G. Evans, "Compositional Control in 3D FGM "; pp. 151-4 in Key Engineering Materials, The Science of Engineering Ceramics III. Trans Tech Publications Inc., Brandrain 6. CH-8707 UetikonZurich, Switzerland, 2005.

${ }^{12}$ S. Yang and J. R. G. Evans, "Acoustic Initiation of Powder Flow in Capillaries," Chem. Eng. Sci., 60, 413-21 (2005).

${ }^{13}$ G. Kiziltas, D. Psychoudakis, J. L. Volakis, and N. Kikuchi, "Topology Design Optimization of Dielectric Substrates for Bandwidth Improvement of a Patch Antenna," IEEE Trans. Antennas Propagation, 51 [10] 2732-43 (2003).

${ }^{14}$ X. Gong, W. H. She, E. Hoppenjans, Z. N. Wing, R. G. Geyer, J. W. Halloran, and W. J. Chappell, "Tailored and Anisotropic Dielectric Constants Through Porosity in Ceramic Components," IEEE Trans. Microwave Theory Tech., 53 [11] 3638-47 (2005).

${ }^{15} \mathrm{G}$. Kiziltas, J. Volakis, Z. Wing, and J. Halloran, "Topology Optimization of a Compact UHF Spiral Array Embedded in a Textured Dielectric"; 6th World Congress on Structural and Multidisciplinary Optimization, Rio de Janeiro, Brazil, June 30-July 3, 2005.

${ }^{16}$ L. W. Coughanour, R. S. Roth, S. Marzullo, and F. E. Sennett, "Solid State Reactions and Dielectric Properties in the System Magnesia-Lime-Tin OxideTitania," J. Res. Natl. Bur. Stand. (U.S.), 54 [3] 149-62 (1955).

${ }^{17}$ L. Favenneca, V. Jousseaumeb, V. Rouessacc, F. Fusalbaa, J. Durandc, and G. Passemarda, "Porous Extreme Low k (ELk) Dielectrics Using a PECVD Porogen Approach," Mater. Sci. Semicond. Process., 7, 277-82 (2004).

${ }^{18}$ S. Seraji, Y. Wu, M. Forbess, S. J. Limmer, T. Chou, and G. Cao, "Sol-GelDerived Mesoporous Silica Films with Low Dielectric Constants," Adv. Mater., 12 [22]: 1695-8 (2000)

${ }^{19}$ Z. N. Wing, B. Wang, and J. W. Halloran, "Permittivity of Porous Titanate Dielectrics," J. Am. Cer. Soc., accepted for publication (JACERS-21327 R1)

${ }^{20}$ J. Yang, A. Sliva, A. Banerjee, R. N. Dave, and R. Pfeffer, "Dry Particle Coating for Improving the Flowability of Cohesive Powders," Powder Tech., 158 [1-3] 21-33 (2005) 